\title{
Effect of dispersion forces on the capillary-wave fluctuations of liquid surfaces
}

\author{
Enrique Chacón, ${ }^{1,3,{ }^{*}}$ Eva M. Fernández, ${ }^{1,2, \dagger}$ and Pedro Tarazona ${ }^{3,4, \dagger}$ \\ ${ }^{1}$ Instituto de Ciencia de Materiales de Madrid, CSIC, 28049 Madrid, Spain \\ ${ }^{2}$ Departamento de Física Fundamental, Universidad Nacional de Educación a Distancia, 28080 Madrid, Spain \\ ${ }^{3}$ Instituto de Ciencia de Materiales Nicolás Cabrera \\ ${ }^{4}$ Departamento de Física Teórica de la Materia Condensada, Condensed Matter Physics Center (IFIMAC), \\ Universidad Autónoma de Madrid, 28049 Madrid, Spain
}

(Received 9 July 2013; revised manuscript received 22 January 2014; published 15 April 2014)

\begin{abstract}
We present molecular dynamics evidence for the nonanalytic effects of the long-range dispersion forces on the capillary waves fluctuations of a Lennard-Jones liquid surface. The results of the intrinsic sampling method, for the analysis of the instantaneous interfacial shape, are obtained in large systems for several cut-off distances of the potential tail, and they show good agreement with the theoretical prediction by Napiórkowski and Dietrich, based on a density functional analysis. The enhancement of the capillary waves is quantified to be within $1 \%$ for a simple liquid near its triple point.
\end{abstract}

DOI: 10.1103/PhysRevE.89.042406

PACS number(s): 68.03.Cd, 05.70.Np, 68.03.Kn

\section{INTRODUCTION}

London dispersion forces are the most ubiquitous attractions among van der Waals molecular interactions, since they are produced by the coupling of fluctuations, even in the absence of permanent electrical dipoles [1], and cannot be avoided, nor screened, in condensed matter bodies. Two molecules at distance $r$ have a long-range (LR) potential energy $\phi_{\mathrm{LR}}(r)=-C / r^{6}$, where the constant $C$ is proportional to the product of their polarizabilities. Derjaguin [2] and Hamaker [3] described the accumulation of these interactions in extended bodies, with a qualitative dependence on the shape of the objects produced by the power-law decay. The phenomenology of complete wetting, capillary condensation, and other interfacial properties of liquids have to incorporate these geometrical effects [4,5]; e.g., the interaction between parallel surfaces separated by a distance $h$ decays as $1 / h^{2}$. This power law becomes an exponential $e^{-h / d}$ for any model system with truncated $\left[\phi(r)=0\right.$, for $\left.r>r_{c}\right]$, or short-range $\left[\phi(r) \propto e^{-\alpha r}\right]$ interactions that includes a molecular distance scale $\left(d \propto r_{c}\right.$ or $\left.d \propto \alpha^{-1}\right)$, in contrast with the scaleless character of the dispersion forces.

The most usual model for molecular interactions is the Lennard-Jones (LJ) potential, $\phi_{\mathrm{LJ}}(r)=4 \epsilon\left[(\sigma / r)^{12}-(\sigma / r)^{6}\right]$. For $r$ well beyond the molecular diameter $\sigma$ the potential has the LR tail from the dispersion forces, with $\epsilon=C /\left(4 \sigma^{6}\right)$ to set the reduced temperature $T^{*}=k T / \epsilon \equiv(\beta \epsilon)^{-1}$. Computer simulations of truncated $\mathrm{LJ}$ systems give properties $X\left(r_{c}\right)$ that depend on the cutoff, and only for $r_{c} \gg \sigma$ do they approach the full effect of the LR interactions as $X^{\mathrm{LR}}-X\left(r_{c}\right) \propto r_{c}^{-s}$. For bulk properties, like the density gap between the coexisting liquid and vapor $\rho_{\mathrm{lv}}\left(r_{c}\right)=\rho_{\mathrm{l}}\left(r_{c}\right)-\rho_{\mathrm{v}}\left(r_{c}\right)$, we expect the exponent $s=3$, which is reduced to 2 for interfacial magnitudes, like the liquid-vapor surface tension $\gamma_{\mathrm{lv}}\left(r_{c}\right)$. Here we use $\mathcal{A}\left(r_{c}\right)=4 \pi^{2} \epsilon\left(\rho_{\mathrm{lv}}\left(r_{c}\right) \sigma^{3}\right)^{2}$ as the relevant Hamaker constant

\footnotetext{
*echacon@icmm.csic.es

$\dagger$ †emfernandez@fisfun.uned.es

†pedro.tarazona@uam.es
}

for the liquid-vapor interface and $\mathcal{A}^{\mathrm{LR}}$ for its value with the density gap of the full $\mathrm{LJ} \rho_{\mathrm{lv}}^{\mathrm{LR}}$.

In this article we present evidence, from molecular dynamics (MD) simulations, of the LR effects on the thermal fluctuations of a liquid surface, predicted by Napiórkowski and Dietrich (ND) 20 years ago [5,6]. The capillary wave theory (CWT) $[7,8]$ uses a continuous description of a liquid surface, as a function $z=\xi(\mathbf{x})$ with $\mathbf{x}$ over the $X Y$ plane, and in terms of its Fourier components $\hat{\xi}_{q}$,

$$
\xi(\mathbf{x})=\hat{\xi}_{0}+\sum_{|\mathbf{q}|>0} \hat{\xi}_{q} e^{i \mathbf{q} \cdot \mathbf{x}} .
$$

As sketched in Fig. 1, any capillary wave (CW) corrugation with wave vector $q$ and small amplitude $\hat{\xi}_{q}$ implies an increase $\Delta A \approx A_{o} q^{2}\left|\hat{\xi}_{q}\right|^{2} / 2$ over the planar cross-sectional area $A_{o}$. The three (instantaneous) configurations in the sketch have equal area $\left(\Delta A / A_{o} \approx 0.2\right)$, and the CWT assumes that they have the same thermodynamic cost, $\gamma_{1 v} \Delta A$, with the macroscopic surface tension $\gamma_{\mathrm{lv}}$. Therefore, in thermal equilibrium the three fluctuations should have the same probability, $\sim e^{-\beta \Delta A \gamma_{\mathbb{v}}}$, as it corresponds to the mean-square amplitude $\left\langle\left|\hat{\xi}_{q}\right|^{2}\right\rangle=\left(\beta \gamma_{\mathrm{lv}} q^{2} A_{o}\right)^{-1}$.

The main effect of these $\mathrm{CW}$ fluctuations is the broadening of the mean density profiles, given by the local displacement of the interface, with mean-square value $\Delta_{\mathrm{CW}}\left(A_{o}\right) \equiv \sum_{q}\left\langle\left|\hat{\xi}_{q}\right|^{2}\right\rangle$, that adds the contributions from each $\mathrm{CW}$ mode (each wave vector q). We made explicit the dependence of $\Delta_{\mathrm{CW}}$ with the transverse cross-section area $A_{o}$ from the lower- $q$ (longwavelength) limit in the CW fluctuations allowed by the lateral size of the system. The right-side column in Fig. 1 gives a molecular view to point that the continuous description of the liquid surface $z=\xi(\mathbf{x})$ cannot be indefinitely extended to larger values of $q$. The classical CWT sets a molecular top of the CW spectrum, $|\mathbf{q}| \leqslant q_{u}$, associated to a wavelength $\Lambda \equiv$ $2 \pi / q_{u}$ in the range of the molecular size $\sigma$. This $\Lambda$ should be considered as an empirical parameter, important to incorporate a qualitative effect (the molecular discreteness), but without major relevance of its precise value, since the sum over the $\mathrm{CW}$ modes (approximated by an integral) leads to a total CW 

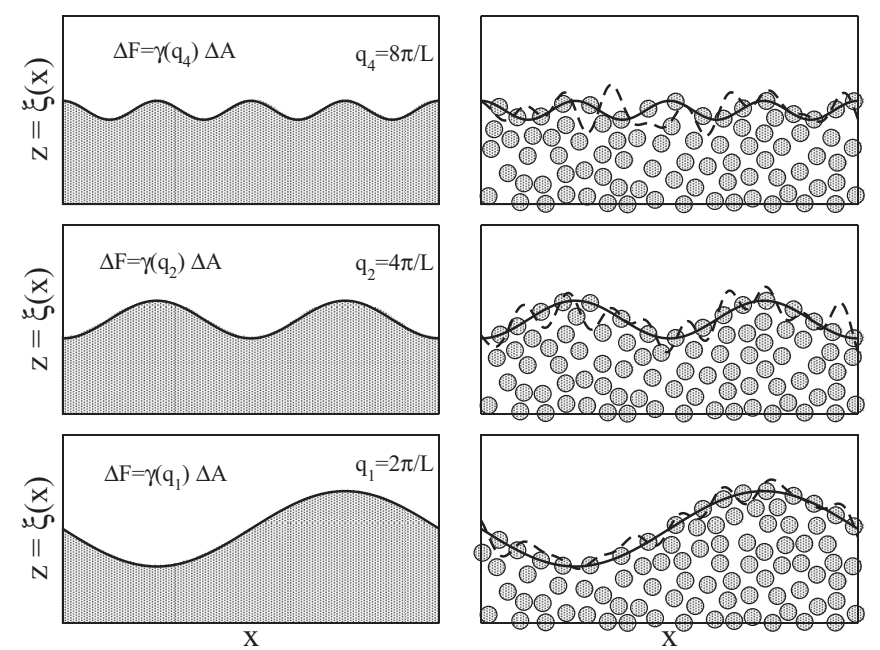

FIG. 1. Sketches of capillary waves on a liquid surface with equal increases of area $\Delta A$ but different wave vectors. The CWT $[7,8]$ assumed equal free energy $\Delta F=\gamma_{\mathrm{lv}} \Delta A$ (i.e., equal probability $\sim e^{-\beta \Delta F}$ ) for the three fluctuations. A bending modulus contribution gives decreasing probabilities from bottom to top, described by increasing values of $\gamma(q)$, in Eq. (3). Napiórkowski and Dietrich predicted enhanced probabilities for intermediate wave vectors. Left: Continuous representation of the instantaneous interface $z=\xi(x)$. Right: Molecular view, in which given molecular positions could be interpreted as different functions $\xi(x)$ : ISM (full line) and GDS (broken line), leading to different functions $\gamma(q)$ except for $\gamma(0)=\gamma_{\mathrm{lv}}$.

mean-square width,

$$
\Delta_{\mathrm{CW}}\left(A_{o}\right) \approx \frac{A_{o}}{(2 \pi)^{2}} \int d^{2} \mathbf{q}\left\langle\left|\hat{\xi}_{q}\right|^{2}\right\rangle=\frac{\log \left(A_{o} / \Lambda^{2}\right)}{4 \pi \beta \gamma_{\mathrm{lv}}},
$$

with a weak logarithmic dependence on $A_{o} / \Lambda^{2}$. Notice that, for simplicity, we are not including gravity effects, which become relevant only for transverse lengths larger than the capillary length, in the range of millimeters for the typical liquid surfaces in the Earth. For larger areas, the inclusion of that contribution cancels the dependence of $\Delta_{\mathrm{CW}}$ with $A_{o}$, and the $\mathrm{CW}$ broadening depends logarithmically on the gravity field [8].

The $\mathrm{x}$-ray dispersion by the $\mathrm{CW}$ at a fluid surface could in principle be used to measure $\left\langle\left|\hat{\xi}_{q}\right|^{2}\right\rangle$, and this experimental access to the contributions from each wave vector suggests that the CWT should be reconsidered. The sharp cutoff of the CW spectrum at $q_{u}$, and the validity of the macroscopic limit for $\left\langle\left|\hat{\xi}_{q}\right|^{2}\right\rangle=\left(\beta \gamma_{\mathrm{lv}} q^{2} A_{o}\right)^{-1}$ for any $q \leqslant q_{u}$, are obvious simplifications of the theory, aimed only to get the global effect on $\Delta_{\mathrm{CW}}$. The molecular limit to the $\mathrm{CW}$ spectrum may be considered with a more physical hypothesis, assuming that, on top of the $\gamma_{\mathrm{lv}} \Delta A$ term, the free energy of a corrugated liquid surface has a term proportional to its mean-square curvature multiplied by a bending rigidity modulus $\kappa[9,10]$. The free energy of a CW mode becomes $\delta F_{q}\left(\hat{\xi}_{q}\right)=\left(\gamma_{\mathrm{lv}} q^{2}+\right.$ $\left.\kappa q^{4}\right)\left|\hat{\xi}_{q}\right|^{2} \mathrm{~A}_{o} / 2+\mathcal{O}\left(\left|\hat{\xi}_{q}\right|^{4}\right)$, so the probability of the surface fluctuations represented in Fig. 1 would be predicted to decrease from bottom to top, rather than to be equal for any $q \leqslant q_{u}$, as assumed in the classical CWT. The total CW square width $\Delta_{\mathrm{CW}}\left(A_{o}\right)$ may now be formally calculated with the sum extended to indefinitely large wave vectors, since their small amplitude will make them irrelevant. In this description, the role of the molecular scale parameter $\Lambda$ in (2) is taken by $2 \pi \sqrt{\kappa / \gamma_{\text {lv }}}$, which has the dimension of a length but, in contrast to $\Lambda$, it does not come as a molecular (high $q$ ) magnitude, but rather it represents a description (or prediction) for the low- $q$ behavior of $\left\langle\left|\hat{\xi}_{q}\right|^{2}\right\rangle$.

It has become customary to define a wave-vector-dependent surface tension as an extension of the $q=0$ limit,

$$
\gamma(q) \equiv\left(\beta\left\langle\left|\hat{\xi}_{q}\right|^{2}\right\rangle q^{2} A_{o}\right)^{-1}=\gamma_{\mathrm{lv}}+\kappa q^{2}+\mathcal{O}\left(q^{4}\right),
$$

to represent the different free energies per unit area of $\mathrm{CW}$ fluctuations like those in Fig. 1, and the surface bending modulus $\kappa$ gives the regular low- $q$ expansion from the thermodynamic surface tension.

Surprisingly, the ND analysis for corrugated surfaces with LR interactions, based on a density functional theory (DFT) for $\gamma(q)$, showed the nonanalytic behavior

$$
\gamma^{\mathrm{LR}}(q)=\gamma_{\mathrm{lv}}^{\mathrm{LR}}+\frac{\mathcal{A}^{\mathrm{LR}}}{32 \pi} q^{2} \log (q b)+\mathcal{O}\left(q^{4}\right),
$$

which implies a negative diverging bending modulus. The LR Hamaker constant depends only on the bulk coexisting densities, and any dependence on the molecular structure of the surface comes through the parameter $b$, which has the dimension of a length. According to this prediction, in a liquid with long-range dispersion forces the equal-area corrugations of the surface sketched in Fig. 1 would show a nonmonotonic probability, as a function of $q$. Over a mesoscopic range $q \approx 0.6 / b$ the $\mathrm{CW}$ fluctuations would be enhanced, both with respect to macroscopic (lower $q$ ) and to microscopic (higher $q$ ) corrugations. The existence of enhanced $\mathrm{CW}$ has been a matter of controversy over the past decade. Claims of observations in X-ray diffraction [11-14], computer simulations [15,16], and theoretical density functional (DF) calculations [17-19] have been answered from different perspectives [10,20,21]. The crucial point is the link between the left and right sides of Fig. 1, i.e., the definition of the continuous surface $z=\xi(\mathbf{x})$, from the instantaneous molecular positions.

In this work we use the intrinsic sampling method (ISM) $[22,23]$ based on an intuitively simple, although computationally expensive, concept. After defining some (optimized) percolation parameters, the molecules within the liquid phase are identified as those belonging to the main percolating cluster, and those on the surface of that cluster are taken as surface pivots. The ISM function $\xi_{\text {ISM }}(\mathbf{x})$ is then (self-consistently) defined to interpolate smoothly between all the pivot positions. The sketches in Fig. 1 give a qualitative representation of the results: When we look at molecular resolution, will find a corrugated surface layer with the molecular centers precisely on the mathematical surface $z=\xi_{\text {ISM }}(\mathbf{x})$. The idea was first proposed by Stillinger [24], but the ISM was its first practical implementation [22,23] followed by other similar approaches [25-30]. The sharp ISM view of the molecular structure at the interface extends the quantitative matching between computer simulations and the $\mathrm{CW}$ theory down to the nanometer size [31] at the high computational cost of a percolation analysis. 
In contrast, other definitions of $\xi(\mathbf{x})$ are computationally much cheaper, in particular, the local Gibbs dividing surface (GDS) defines the local position $z=\xi_{\mathrm{GDS}}(\mathbf{x})$ from the total number of molecules within a narrow prism across the interface. The $q=0$ component, $\hat{\xi}_{0}$, gives the usual Gibbs dividing plane such that the volume below that plane multiplied by the density of the liquid bulk plus the volume above it multiplied by the vapor density matches precisely the total number of particles in the system. For the extension to a corrugated surface we have to define the width of the interfacial slab, $-\ell_{z} \leqslant z \leqslant \ell_{z}$, and then the Fourier components with $q \neq 0$ are given by

$$
\hat{\xi}_{q}=\frac{1}{A_{o} \rho_{\mathrm{lv}}} \sum_{-\ell_{z} \leqslant z_{i} \leqslant \ell_{z}} e^{i \mathbf{q} \cdot \mathbf{x}_{i}}
$$

where the sum runs over all the molecules within the slab. This is a one-particle definition of $\xi(\mathbf{x})$, i.e., a linear application of the density operator $\hat{\rho}(\mathbf{r})=\sum_{i} \delta\left(\mathbf{r}-\mathbf{r}_{i}\right)$, while the ISM percolation analysis requires the $N$-particle distribution. The local GDS definition for the mean-square amplitude of the CW fluctuation, $\left\langle\left|\hat{\xi}_{q}\right|^{2}\right\rangle$, is a two-particle magnitude, proportional to the integral of the structure factor over the interfacial slab and therefore directly related to the experimental study of the $\mathrm{CW}$ fluctuations with the $\mathrm{x}$-ray dispersion. The results for the molecular positions in Fig. 1 (using the whole plotted region as interfacial slab) are shown by the broken lines in the right-side frames. For the lowest one the molecular configuration, chosen to give a pure $\mathrm{CW}$ undulation with the ISM, gives a slightly rougher interface when we extract $z=\xi_{\mathrm{GDS}}(\mathbf{x})$ from (5), but the amplitude of the main Fourier component $(q \sigma=0.05$ in this case) is similar with both definitions. Indeed, any reasonable definition, explicit, as the ISM and the GDS in computer simulations [16], in the analysis of surface diffraction experiments [10,12], or the implicit DFT analysis of CW fluctuations [4,17], gives $\gamma_{1 \mathrm{v}}$ from the $q \sigma \ll 1$ limit of (3).

However, looking at the top-right frame in Fig. 1 we observe the qualitative problem that has encumbered the observation of the ND effect. The GDS cannot separate density fluctuations near the surface from the surface fluctuation described by the CWT. In the GDS description of any given molecular configuration the instantaneous height of the intrinsic surface $z=\xi_{\text {GDS }}(\mathbf{x})$ may be locally shifted up or down by the presence of small bulklike fluctuations within the interfacial slab, so even if we select along a MD simulations the configurations with a perfectly flat surface layer [i.e., $\xi_{\text {ISM }}(\mathbf{x})=0$ ] we would observe corrugations in $\xi_{\mathrm{GDS}}(\mathbf{x})$. The problem becomes increasingly important for the higher wave vectors, as far as making the formal definition of the GDS surface fully unrelated to the actual corrugations of interface at molecular scale. The result is that the coefficient $\kappa$, from the $q^{2}$ term in (3), may change wildly with the definition of $\xi(\mathbf{x})[18,19]$. In fact, the one-particle methods cited above give a negative bending modulus, irrespective of the (long or short) range of the interactions. At large $q$ the (so-defined) mean-square amplitude $\left\langle\left|\hat{\xi}_{q}\right|^{2}\right\rangle$ does not even decay as $q^{-2}$, so $\gamma(q)$ obtained from (3) has a fully unphysical decreasing shape, missing any molecular top in the $\mathrm{CW}$ spectrum. Therefore, any attempt to test the theoretical prediction (4) from experiments or computer simulations would be frustrated by the fact that $\gamma(q)$ becomes a monotonously decreasing function (even for models with strictly short-range interactions). The subtraction of the bulk signal to $\left\langle\left|\hat{\xi}_{q}\right|^{2}\right\rangle$ may provide a positive bending modulus [32,33], but $\kappa$ would depend on the precise way in which that bulk contribution is defined, which makes it very difficult to discern the subtle logarithmic divergence (4). In contrast, all the reported ISM results for truncated or short-range interactions show increasing functions $\gamma(q)$, with the bending modulus $0.2 \lesssim \beta \kappa \lesssim 1$. Moreover, since the surface $\xi_{\text {ISM }}(\mathbf{x})$ is defined to be smooth at a molecular scale, the method gives a function $\gamma(q)$ that increases even faster from the higher-order terms in (3).

The aim of the present article is to use the ISM, as a quantitatively accurate link between CWT and computer simulations, to explore the long-range effects of dispersion forces (4), predicted by Napiórkowski and Dietrich through a mean-field analysis [6]. The question is whether the nonanalytic behavior of $\gamma(q)$ is a robust characteristic of the $\mathrm{CW}$ fluctuations in liquid surfaces that should come consistently from the two-particle DF description or from any many-particle percolative analysis. Also, it would be important to quantify the long-range effects, relative to the total result for $\gamma(q)$, to figure out under which conditions (system size and wave-vector range) we may expect to observe its effects in a realistic model of liquid surface. Since any computer simulation has to be done with the LJ tails truncated at some $r_{c}$ cut-off distance, the plan of our work is obtain the MD-ISM simulation results for the wave-vector-dependent function $\gamma\left(q, r_{c}\right)=\gamma_{\mathrm{lv}}\left(r_{c}\right)+\kappa\left(r_{c}\right) q^{2}+\mathcal{O}\left(q^{4}\right)$ with different cut-off distances and to add to them the theoretical CWT-DFT predictions from the contribution of the LR tail to reconstruct the $r_{c} \rightarrow \infty$ limit for $\gamma^{\mathrm{LR}}(q)$.

\section{MEAN-FIELD DENSITY FUNCTIONAL THEORY}

The simplest theoretical framework to study the dependence of $\gamma\left(q, r_{c}\right)$ with the cutoff of the LJ tail is the CWT-DFT connection proposed by Dietrich and coworkers [6,17], where the mean-field density functional (MF-DF) approximation to the cost to corrugate the surface is given by the following:

$$
\begin{aligned}
F_{\text {sur }}[\xi(\mathbf{R})] & \equiv \mathcal{F}[\rho(z-\xi(\mathbf{R}))]-\mathcal{F}[\rho(z)] \\
& =\sum_{q} A_{o} q^{2} \gamma_{\mathrm{DF}}(q)\left|\hat{\xi}_{q}\right|^{2} / 2+\mathcal{O}\left(\hat{\xi}_{q}^{4}\right) .
\end{aligned}
$$

Within this mean-field approximation the free energy needed to corrugate the surface in a short-ranged (SR) system, with truncated interactions at $r=r_{c}$, differs from that with the full long-ranged (LR) dispersion tail $\left(r_{c} \rightarrow \infty\right)$ by the following:

$$
\begin{aligned}
\Delta F_{\mathrm{sur}}\left[\xi ; r_{c}\right] \equiv & F_{\text {sur }}^{\mathrm{SR}}\left[\xi ; r_{c}\right]-F_{\mathrm{sur}}^{\mathrm{LR}}[\xi] \\
= & \frac{1}{2} \int d \mathbf{r}_{1} \int d \mathbf{r}_{2}\left\{\rho\left[z_{1}-\xi\left(\mathbf{R}_{1}\right)\right] \rho\left[z_{2}-\xi\left(\mathbf{R}_{2}\right)\right]\right. \\
& \left.-\rho\left(z_{1}\right) \rho\left(z_{2}\right)\right\} \Delta \phi_{r_{c}}\left(r_{12}\right)
\end{aligned}
$$

with

$$
\Delta \phi_{r_{c}}(r) \equiv \phi^{\mathrm{SR}}(r)-\phi^{\mathrm{LR}}(r)=\left\{\begin{array}{ll}
0 & r<r_{c} \\
\frac{4 \epsilon}{(r / \sigma)^{6}} & r>r_{c}
\end{array} .\right.
$$


The leading contribution to $\Delta F_{\text {sur }}\left[\xi ; r_{c}\right]$ is given by the kink approximation for the density profile, i.e., taking the liquid bulk density, $\rho(z)=\rho_{l}$, for any $z \leqslant 0$ and the vapor density, $\rho(z)=\rho_{v}$, for $z>0$. The result is as follows:

$$
\begin{aligned}
\Delta F_{\mathrm{sur}}\left[\xi ; r_{c}\right]= & -\frac{\rho_{\mathrm{lv}}^{2}}{2}\left[\int_{z_{1}<\xi\left(\mathbf{R}_{1}\right)} d \mathbf{r}_{1} \int_{z_{2}>\xi\left(\mathbf{R}_{2}\right)} d \mathbf{r}_{2}\right. \\
& \left.\Delta \phi_{r_{c}}\left(r_{12}\right)-\int_{z_{1}<0} d \mathbf{r}_{1} \int_{z_{2}>0} d \mathbf{r}_{2} \Delta \phi_{r_{c}}\left(r_{12}\right)\right] .
\end{aligned}
$$

The result was obtained [6] by ND [see Eqs. (4.2) and (4.3) there]. We present the details in the Supplemental Material [34] in order to make clear the approximations involved. The expression obtained is as follows:

$$
\Delta F_{\mathrm{sur}}\left[\xi ; r_{c}\right]=-\frac{A}{2} \frac{\pi \epsilon \rho_{\mathrm{lv}}^{2} \sigma^{6}}{r_{c}^{2}} \sum_{q}\left|\xi_{q}\right|^{2} q^{2} \chi\left(q r_{c}\right)
$$

with the function

$$
\chi(x) \equiv 8 x^{2} \int_{x}^{\infty} d y \frac{1-J_{0}(y)}{y^{5}} .
$$

The comparison of (10) with (6), up to order $\left|\hat{\xi}_{q}\right|^{2}$ for each wave vector $q$, gives the predicted difference between $\gamma\left(q ; r_{c}\right)$ for the simulated systems with cutoff $r_{c}$ and $\gamma^{\mathrm{LR}}(q)$ for a system with the full dispersion potential,

$$
\gamma^{\mathrm{LR}}(q)=\gamma\left(q ; r_{c}\right)+\frac{\mathcal{A}\left(r_{c}\right)}{4 \pi r_{c}^{2}} \chi\left(q r_{c}\right)+\mathcal{O}\left(r_{c}^{-3}\right) .
$$

The lines in Fig. 2 show the main features of $\chi(x)$. The ND effect comes from the nonanalytic dependence of the Bessel function integral in the limit $x \rightarrow 0$,

$$
\chi(x) \approx 1+\frac{x^{2}}{8} \log \left(\frac{x}{5.03}\right)+\mathcal{O}\left(x^{4}\right),
$$

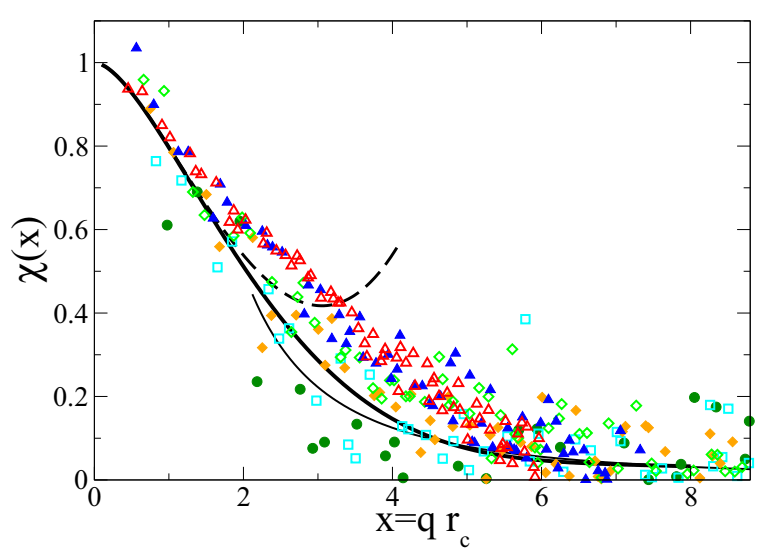

FIG. 2. (Color online) The DF-CWT function $\chi(x)$ (thick line), its expansions (13) for $x \ll 1$ (broken line), and $\chi(x) \approx 2 / x^{2}$ for $x \gg 1$ (thin line). Symbols: MD-ISM results with $\gamma^{\mathrm{LR}}(q)$ from Fig. 4(c) used to get $\left[\gamma^{\mathrm{LR}}(q)-\gamma\left(q ; r_{c}\right)\right] /\left[\gamma_{\mathrm{lv}}^{\mathrm{LR}}-\gamma_{\mathrm{lv}}\left(r_{c}\right)\right]$ for $r_{c} / \sigma=$ 3.019 [empty up-triangles (red)], 3.75 [full up-triangles (blue)], 4.4 [empty diamonds (light green)], 5.0 [full diamonds (orange)], 5.5 [empty squares (cyan)], and 6.5 [full circles (dark green)]. with the coefficient $2 e^{\frac{3}{2}-C} \approx 5.03$ in terms of the EulerMascheroni constant $C$. If we replace (13) in (12) we recover ND equation (4). Figure 2 shows that the expansion (13) is a good approximation for $x \lesssim 2$, but for large $x$ the function decays as $\chi(x) \approx 2 / x^{2}$.

It is worth pointing out that the nonanalytic behavior (4) of $\gamma^{\mathrm{LR}}(q)$ has the same origin as the $S(q) \sim q^{3}$ dependence predicted for the structure factor of a bulk liquid with LR interactions [35]. The LR interactions appear as a tail in the direct correlation function of the bulk fluid, $c(r) \approx$ $-\beta \phi^{\mathrm{LR}}(r) \approx \beta C / r^{6}$, for $r \gg \sigma$. This behavior is captured by the MF-DF approximation used in (7), and it is reflected both in the $q \sigma \ll 1$ behavior of the bulk density fluctuations $\left[S(q) \sim q^{3}\right.$, from the radial symmetry] and in the CW fluctuations of the interface $\left[\gamma(q) \sim q^{2} \log (q)\right.$, from the planar symmetry].

For $q r_{c} \gg 1$ the main effect of the dispersion forces on the mean-square amplitude of the CW fluctuations, i.e., on $\gamma(q)$, comes from the change in the coexisting bulk densities rather than from the direct geometrical effects of the $1 / r^{6}$ tail over the corrugated surface. Therefore, it is useful to separate the bulk density effect in $\rho_{\mathrm{lv}}\left(r_{c}\right)$ from the specific surface effects of long-range interactions. For that we define the function $\frac{\gamma\left(q ; r_{c}\right)}{\mathcal{A}\left(r_{c}\right)}$, and its $\operatorname{LR}\left(r_{c} \rightarrow \infty\right)$ limit $\frac{\gamma^{\mathrm{LR}}(q)}{\mathcal{A}^{\mathrm{LR}}}$, related by the following:

$$
\frac{\gamma^{\mathrm{LR}}(q)}{\mathcal{A}^{\mathrm{LR}}}=\frac{\gamma\left(q ; r_{c}\right)}{\mathcal{A}\left(r_{c}\right)}+\frac{\chi\left(q r_{c}\right)}{4 \pi r_{c}^{2}}+\mathcal{O}\left(r_{c}^{-4}\right) .
$$

The $q=0$ limit gives the theoretical prediction for the behavior of the macroscopic surface tension versus $r_{c}$,

$$
\frac{\gamma_{\mathrm{lv}}\left(r_{c}\right) \sigma^{2}}{\mathcal{A}\left(r_{c}\right)} \approx \frac{\gamma_{\mathrm{lv}}^{\mathrm{LR}} \sigma^{2}}{\mathcal{A}^{\mathrm{LR}}}-\frac{\sigma^{2}}{4 \pi r_{c}^{2}}+\mathcal{O}\left(r_{c}^{-4}\right) .
$$

\section{MOLECULAR DYNAMICS SIMULATIONS}

We have used the software package LAMMPS [36] to run $N V T$ MD simulations of a LJ liquid slab with cut-off distances from $r_{c}=2.5 \sigma$ to $6.5 \sigma$ at $k_{B} T / \epsilon=T^{*}=0.763$ close to the triple temperature. The $\mathrm{LJ}$ potentials are not shifted to zero at the cut-off distance, and we use the LJ parameters as units of distance $(\sigma)$ and energy $(\epsilon)$. Our systems consist of a liquid slab surrounded by vapor, with 176441 particles in a large simulation box with $L_{x}=L_{y}=41.83 \sigma$ and $L_{z}=250 \sigma$, to explore $\mathrm{CW}$ fluctuations down to $q=2 \pi / L_{x}=0.15 / \sigma$. The large thickness of the liquid slab (approximately $3 L_{x}$ ) is to avoid the coupling between the fluctuations on the two interfaces, even for the lowest $q$ and the largest $r_{c}$. The slow $\mathrm{CW}$ dynamics requires also very long MD times for the ISM analysis. For each $r_{c}$ we used $2 \times 10^{6}$ MD steps to equilibrate the system before sampling 5000 configurations of the liquid slab, separated by $5000 \mathrm{MD}$ time steps, $d t=$ $5 \times 10^{-3} \sigma \sqrt{m / \epsilon}$.

\section{A. Surface thermodynamic}

To get an accurate measure of the effects of a long-range (LR) potential on the capillary waves (CW) fluctuations we have to quantify the effects on the bulk coexisting densities for 


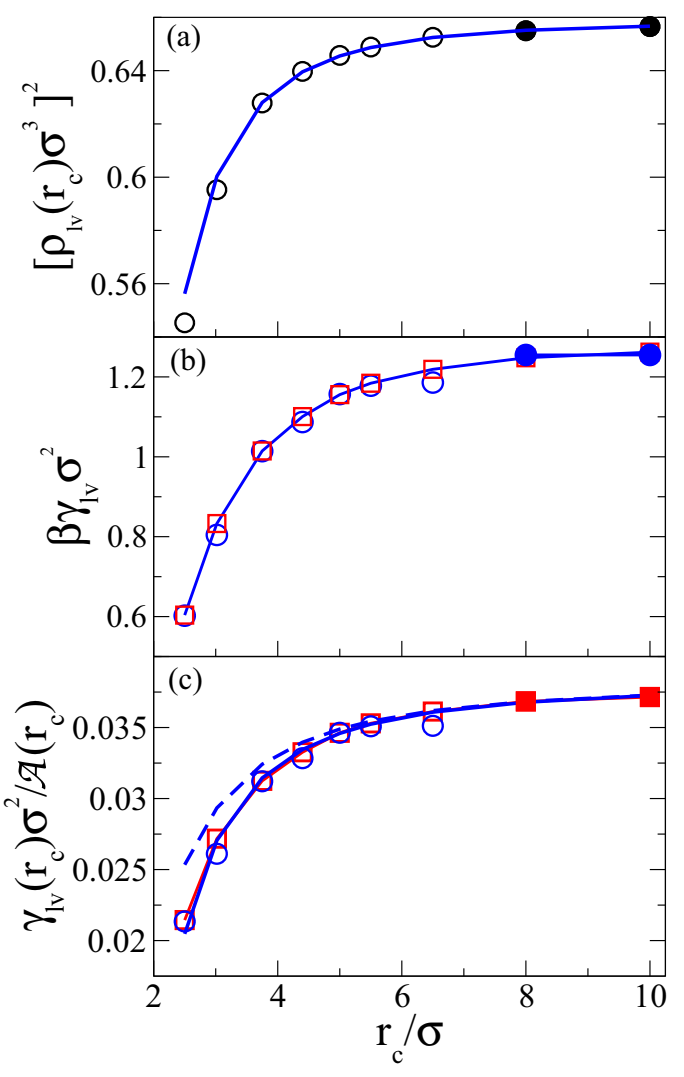

FIG. 3. (Color online) Thermodynamic simulation results for the LJ fluid at $k_{B} T / \epsilon=0.763$ as a function of cut-off distance $r_{c}$. (a) The square of the density gap between the coexisting liquid and vapor, $\rho_{\mathrm{lv}}\left(r_{c}\right)$, as function of cut-off distance $r_{c}$. The full line is the fit $\left[\rho_{\mathrm{lv}}\left(r_{c}\right)\right]^{2}=0.658-1.594\left(r_{c} / \sigma\right)^{-3}$ over the range $3.5 \leqslant r_{c} / \sigma \leqslant$ 10.0. (b) Adimensional surface tension $\beta \gamma_{\mathrm{lv}} \sigma^{2}$. The circles show the values obtained from the pressure tensor route, and the squares show from the best fits to $\gamma\left(q ; r_{c}\right)=\gamma_{\mathrm{lv}}\left(r_{c}\right)+\kappa\left(r_{c}\right) q^{2}$ over the wave-vector range $q \sigma \leqslant 0.7$. The line is a guide to the eye. (c) The symbols show $\gamma_{\mathrm{lv}}\left(r_{c}\right) \sigma^{2} / \mathcal{A}\left(r_{c}\right)$ as function of $r_{c}$. The dashed (red) line is the mean-field prediction to lowest order on $r_{c}^{-2}$ given by Eq. (15), with $\gamma_{\mathrm{lv}}^{\mathrm{LR}} \sigma^{2} / \mathcal{A}^{\mathrm{LR}}=0.038$. The full (blue) line is the fit including a higherorder term, $\frac{\gamma_{\mathrm{v}}\left(r_{c}\right) \sigma^{2}}{\mathcal{A}\left(r_{c}\right)}=0.038-\frac{\sigma^{2}}{4 \pi r_{c}^{2}}-\frac{0.19}{\left(r_{c} / \sigma\right)^{4}}$. For completeness, in the three panels, the full symbols show results obtained with an smaller simulation box, $L_{x}=L_{y}=20.91 \sigma$ and 24325 particles.

the liquid $\left(\rho_{l}\right)$ and vapor $\left(\rho_{v}\right)$ phases. Since we keep the same temperature for all the simulations, the systems with longer dispersion tails show higher $\rho_{l}$ and lower $\rho_{v}$. The density gap $\rho_{\mathrm{lv}}$, in Fig. 3(a), shows a $8 \%$ increase between $r_{c}=2.5 \sigma$ and the extrapolation to the LR limit.

We have used the virial expression to get the thermal average of the pressure tensor components over the whole volume $V=A_{o} L_{z}$,

$$
P_{\alpha \beta} V=\left\langle\sum_{i=1}^{N} m_{i} \mathbf{v}_{i, \alpha} \mathbf{v}_{i, \beta}+\sum_{i=1}^{N-1} \sum_{j>i}^{N} \mathbf{r}_{i j, \alpha} \mathbf{f}_{i j, \beta}\right\rangle,
$$

with $\alpha$ and $\beta$ the Cartesian coordinates $(x, y, z)$, with $\mathbf{v}_{i, \alpha}$ being the velocity of particle $i$ in direction $\alpha, \mathbf{r}_{i j}$ the vector joining atoms $i$ and $j$, and $\mathbf{f}_{i j}$ the force between these two atoms. The surface tension of the liquid-vapor interfaces was obtained as

$$
\gamma_{\mathrm{lv}}=L_{z}\left[P_{z z}-\frac{1}{2}\left(P_{x x}+P_{y y}\right)\right] .
$$

As expected, the function $\gamma_{\mathrm{lv}}\left(r_{c}\right)$, in Fig. 3(b), increases monotonically, since the interactions with longer range imply lower ratio $T / T_{c}\left(r_{c}\right)$ to the critical temperature and larger density gap $\rho_{\mathrm{lv}}\left(r_{c}\right)$.

The first evidence that the DF-CWT theory properly describes these systems comes from the $r_{c}$ dependence of the macroscopic surface tension. The MD simulation results for $\gamma_{\mathrm{lv}}\left(r_{c}\right) \sigma^{2} / \mathcal{A}\left(r_{c}\right)$ presented in Fig. 3(c), from those for $\rho_{\mathrm{lv}}\left(r_{c}\right)$ and $\gamma_{\mathrm{lv}}\left(r_{c}\right)$ in Figs. 3(a) and 3(b), give very good agreement with (15) for any $r_{c} \gtrsim 4 \sigma$ and provide the value $\gamma_{\mathrm{lv}}^{\mathrm{LR}} \sigma^{2} / \mathcal{A}^{\mathrm{LR}}=0.038$ for the (extrapolated) system with the full LR dispersion forces. So our estimation of the surface tension for a (full range) $\mathrm{LJ}$ fluid at $T^{*}=0.763$, is $\beta \gamma_{\mathrm{lv}}^{\mathrm{LR}} \sigma^{2} \approx 1.29$, in good agreement with reported results [37]. For systems with shorter tails $\left(r_{c} \lesssim 4 \sigma\right)$ the simulation results show the presence of a next-to-leading correction, proportional to $1 / r_{c}^{4}$ that would arise if we go beyond the simplest kink approximation for the density profile used in (10).

\section{B. The intrinsic sampling method}

The ISM is an operational procedure to define, from the molecular positions, the intrinsic surface, $z=\xi(\mathbf{x})$, that represents the instantaneous boundary between the liquid and the vapor phases. The method is based on the percolation analysis of the liquid slab and it has been extensively tested over the past decade in the $\mathrm{CW}$ analysis of simple and complex fluid surfaces $[22,23,38]$ and more recently for the fluctuations of adsorbed films [39]. A detailed account of the method and its applications has been presented in Refs. [22,23,38], the latter being the slight modification of the original procedure which is used in the present work.

With its Fourier components restricted to wave vectors $|\mathbf{q}| \leqslant q_{m} \equiv 2 \pi / \sigma$, the intrinsic surface at the maximum level of resolution is defined to have the minimum possible area, but going exactly through all the particles selected to represent the outer liquid layer. To choose the particles in that layer, for each configuration of the liquid slab, we first use a percolation analysis to eliminate any particle with less that three neighbors at a distance below $1.5 \sigma$. An initial set of surface pivots is selected as the most external molecules in each of the prisms arranged in a $22 \times 22$ mesh (bin size $\approx 4 \sigma^{2}$ ), and the initial intrinsic surface is the minimal area surface going through these $N_{0}=22^{2}$ initial pivot particles. Then we incorporate the particle which is closest to the minimal surface as a new surface pivot and recalculate the new intrinsic surface $\xi(\mathbf{x})$ to go through all the previously selected pivots plus the new one. This procedure is iterated until we reach a target occupation of the first layer $n_{s}=N_{0} / A_{0}$, which is the essential control parameter in the procedure [38]. Now the problem is to fix the optimum value of the control parameter $n_{s}$ or, equivalently, to discern what is the occupation of the outer liquid layer, $n_{s}$, which gives the best description of the interface at molecular level. The optimal value of $n_{s}$ may be consistently determined 
from a wide range of structural and dynamical properties of the interface [40]. In our case we have used the structural criterion which is based on the analysis of the intrinsic density profiles for each occupation and discarding the occupations with unphysical behavior of its intrinsic density profile. With this criterion we have obtained an optimal occupation of $n_{s} \sigma^{2}=0.75$ for the systems analyzed here.

Small changes in the parameter $n_{s}$ should be accepted as reasonable variants in the definition of the intrinsic surface. Moreover, even for a given choice of these surface pivots, the only physical reality behind the liquid surface are the molecular positions and we could use different continuous functions $z=\xi(\mathbf{x})$ that interpolate between those molecular positions. In particular, instead of the minimal area surface (with $q \leqslant 2 \pi / \sigma)$ used in the original ISM, we have used here a faceted surface from a two-dimensional Delaunay triangulation (DT). The DT is performed using the pivots coordinates in the plane $\mathbf{x}=(x, y)$. With the DT we identify the nearest neighbors of each pivot. This information is then used to construct a three-dimensional triangulated surface, where the triangles edges join each pivot to its nearest neighbors. Then the Fourier components of $\xi(\mathbf{x})$ are obtained by fitting the Delaunay triangulated surface to the Fourier expansion (11). The procedure and the difference in the results with the minimal area construction are discussed in Ref. [40] and in the Supplemental Material [34].

Our operational definition of the intrinsic surface gives us direct access to all the Fourier components $\hat{\xi}_{\mathbf{q}}$ for each analyzed molecular configurations. The average along the MD simulation gives the mean-square $\mathrm{CW}$ amplitude $\left\langle\left|\hat{\xi}_{q}\right|^{2}\right\rangle$, and from (3) we get directly the $q$-dependent surface tension $\gamma\left(q, r_{c}\right)$.

\section{RESULTS AND DISCUSSION}

The MD-ISM functions $\gamma\left(q ; r_{c}\right)$ in Fig. 4(a) are similar to previous ISM results for truncated or short-range potentials. They are monotonically increasing and extrapolate to a value $\gamma\left(0 ; r_{c}\right)$, in very good agreement with the independent results for $\gamma_{\mathrm{lv}}\left(r_{c}\right)$ from the integral of the pressure tensor. As we can see in the inset of Fig. 4(a), the study of the low- $q$ limit of the function $\gamma\left(q ; r_{c}\right)$ provides another signature of the ND singular behavior (4), namely that the fits of $\gamma\left(q ; r_{c}\right)$ to the regular expansion (3) give slightly decreasing bending modulus $\kappa\left(r_{c}\right)$ when we increase $r_{c}$. However, $\kappa\left(r_{c}\right)$ is never negative and in order to verify the nonanalytical behavior of $\gamma\left(q ; r_{c}\right)$ it becomes necessary to analyze whole range of $q$.

Our first concern in comparing with the MD-ISM results for $\gamma\left(q ; r_{c}\right)$ is to take out the dependence $\gamma\left(q ; r_{c}\right) \propto \Delta \rho_{\mathrm{lv}}^{2}$ from the change in the bulk coexisting phases that gives the main correction $\sim r_{c}^{-3}$ to (12). We expect a much better convergence for the expansion (14) with the two contributions presented in the main panel and inset of Fig. 4(b). The collapse of the $\gamma\left(q ; r_{c}\right) / \mathcal{A}\left(r_{c}\right)$ lines for large $q$ (main panel) is consistent with the rapid decay of $\chi(x) \approx 2 / x^{2}$ for $x \gtrsim 2$, as shown in Fig. 2 . The scaling $\chi\left(q r_{c}\right)$ on the horizontal axis and the term $\propto 1 / r_{c}^{2}$ makes the LR correction very small for the largest $r_{c}$ and fully irrelevant unless we have access to very low $q$ corrugations of the liquid surface. The extrapolation of the MD-ISM results to
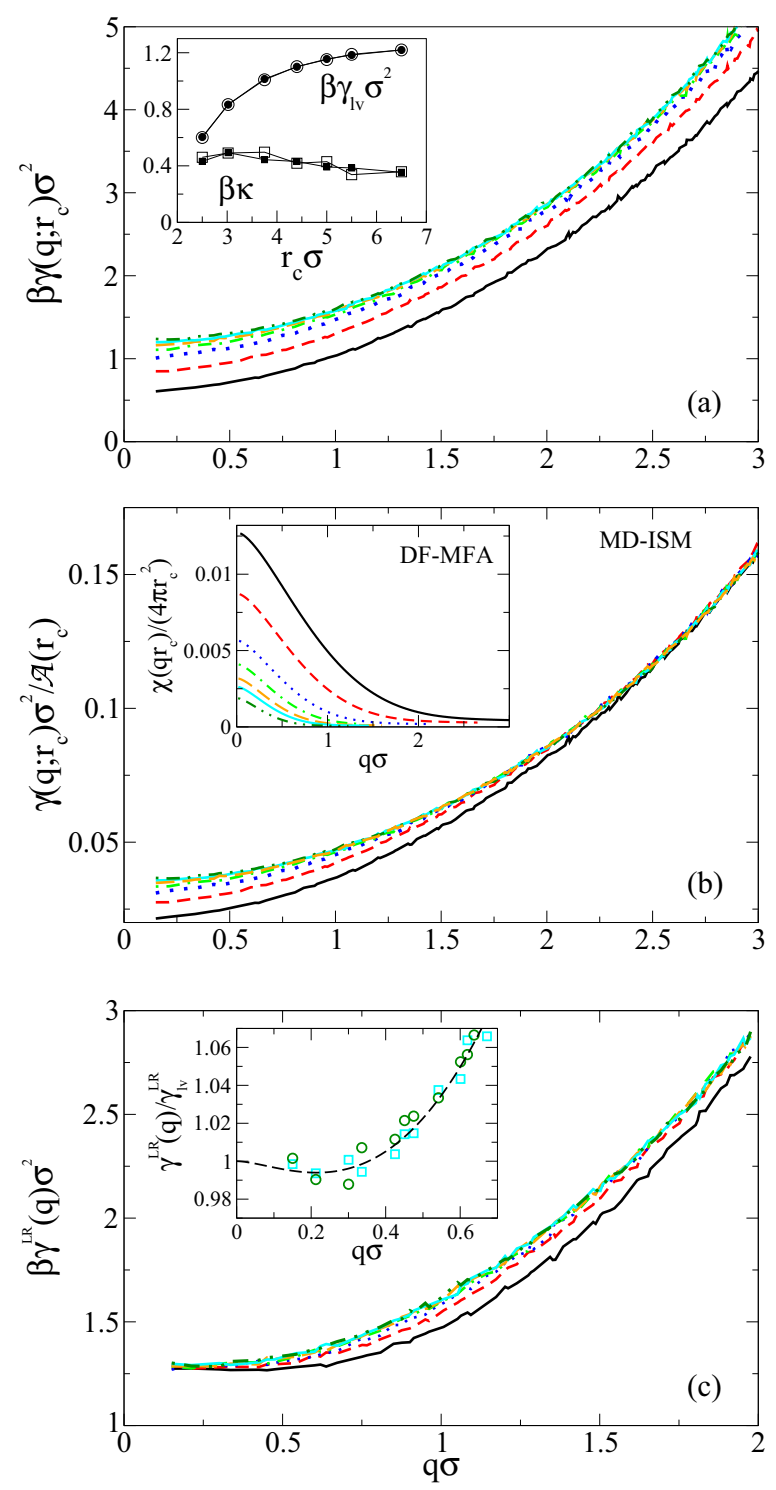

FIG. 4. (Color online) In the three panels the lines present the results for $r_{c} / \sigma=2.5$ [dark solid (black) line], 3.019 [short dashed (red) line], 3.75 [dotted (blue) line], 4.4 [dashed-dotted (light green) line], 5.0 [large dashed (orange) line], 5.5 [light solid (cyan) line], and 6.5 [dashed-double dotted (dark green) line]. (a) The ISM wavevector-dependent surface tension $\beta \gamma\left(q ; r_{c}\right) \sigma^{2}$. Inset: The surface tension $\beta \gamma_{\mathrm{lv}}\left(r_{c}\right) \sigma^{2}$ (circles) and the bending modulus $\beta \kappa$ (squares) from the best fits to $\gamma\left(q ; r_{c}\right)=\gamma_{\mathrm{lv}}\left(r_{c}\right)+\kappa\left(r_{c}\right) q^{2}$ over the wave-vector range $q \sigma \leqslant 0.5$ (full black symbols) and $q \sigma \leqslant 0.7$ (open symbols). (b) The simulation MD-ISM results for $\gamma\left(q ; r_{c}\right) \sigma^{2} / \mathcal{A}\left(r_{c}\right)$ (see text) and the theoretical DF-MFA prediction (inset) for the long-range tail Eq. (14). (c) The prediction for the full long-range result $\beta \gamma^{\mathrm{LR}}(q) \sigma^{2}$ adding the MD-ISM and the DF-MFA contributions presented in panel (b). The inset gives a detailed view of the results for $r_{c} / \sigma=5.5$ (squares) and 6.5 (circles), together with their fit to the non-analytic form (4), with $b / \sigma \approx 2.5$.

$q=0$ is consistent with the virial results for $\gamma_{\mathrm{lv}}\left(r_{c}\right) / \mathcal{A}\left(r_{c}\right)$ as discussed above.

Figure 4(c) uses the sum of the two contributions in Fig. 4(b) to get our estimate for $\gamma^{\mathrm{LR}}(q)$ from the simulations 
of each truncated system. The curve for $r_{c}=2.5 \sigma$ is clearly below those for larger $r_{c}$, reflecting that the matching between what is included in the simulations and what is added from the theoretical approximation cannot be perfect for a very short cutoff. Increasing $r_{c}$ we observe that the different curves collapse within the inherent noise of the simulation results. Despite the strong theoretical simplifications of the MF-DF and the sharp-kink density profile, we get a quantitatively consistent correction for the MD-ISM results for any $r_{c} \gtrsim$ $4.4 \sigma$. Their common prediction for $\gamma^{\mathrm{LR}}(q) / \mathcal{A}^{\mathrm{LR}}$ with the full LR interactions [inset in Fig. 4(c)] is in good agreement with the predicted nonanalytic behavior (4). The only fitting parameter, $b / \sigma \approx 2.5 \pm 0.3$, determines the analytic part of $\gamma^{\mathrm{LR}}(q)$ from the short-range structure, the LJ liquid surface, as extracted by the MD-ISM for $T^{*}=0.763$.

In Fig. 2 we present complementary evidence for the ND effect. Once we have $\gamma^{\mathrm{LR}}(q)$ we may compare $\left[\gamma^{\mathrm{LR}}(q)-\right.$ $\left.\gamma\left(q ; r_{c}\right)\right] /\left[\gamma_{\mathrm{lv}}^{\mathrm{LR}}-\gamma_{\mathrm{lv}}\left(r_{c}\right)\right]$, for each $r_{c}$, with the theoretical function $\chi\left(q r_{c}\right)$. The relatively large noise in the simulation results (despite the computational effort done in our MD simulations) reflects that we are extracting a very subtle effect through the amplification of the smooth curves $\gamma\left(q ; r_{c}\right)$ in Fig. 4(a), but still the evidence for the ND effect is clear.

As noted above, the shape of $\gamma\left(q ; r_{c}\right)$ depends strongly on the definition of the intrinsic surface. Even within the ISM procedure there is some weak dependence on the parameters used in the ISM percolation-interpolation analysis. These parameters have been optimized with regard to structural, kinetic, and dynamical properties of the liquid surface [40], but still there is always some uncertainty. In the Supplemental Material [34] we compare with the results of another two interpolation recipes, which have been successfully used in the investigation of the structure of liquid interfaces [40] and biological membranes [41]. As it should be expected, the thermodynamic limit $\gamma\left(0, r_{c}\right)=\gamma_{\mathrm{lv}}\left(r_{c}\right)$ is robust with respect to the particular definition of $\xi(\mathbf{x})$ and indeed the extrapolations to $q=0$ of the ISM for $\gamma\left(q, r_{c}\right)$ show differences of less than $0.2 \%$ between different interpolation schemes. These are fully indistinguishable in the scale of Fig. 3(c) and of the inset of the Fig. 4(a), and they are perfectly compatible with the error bars in the MD simulations. On the contrary, the values of $\gamma\left(q, r_{c}\right)$ for $q>0$ present small but visible differences, which may be appreciated in the quadratic term $\kappa$, which is known to depend on the specific choice used in the definition of the intrinsic surface $\xi(\mathbf{x})$ [19]. In particular, GDS definitions of the intrinsic surface lead to a negative surface bending modulus [23], although recent results [32] show that with a careful subtraction of the bulk contribution the sign of $\kappa\left(r_{c}\right)$ for a truncated LJ liquid may become positive at higher temperatures.

Within the ISM choices the changes in $\beta \kappa\left(r_{c}\right)$ are small and they represent rigid shifts of about \pm 0.1 with respect to those presented in the inset of Fig. 4(a). Therefore, the variation of the bending modulus with respect to the cut-off distance is more robust than its asymptotic LR value [34]. This result is similar to that obtained in the study of the capillary wave spectra of adsorbed liquid films, where the quadratic contribution to the dependence of the $\mathrm{CW}$ spectrum with the width of the liquid film can be perfectly evaluated with our procedure, although the quadratic behavior of the free surface (film width infinity) depends on the kind of intrinsic surface used [39,42]. The change of $\beta \kappa\left(r_{c}\right)$ by \pm 0.1 would represent a $\pm 30 \%$ change in our estimation for $b$, clearly beyond the error bar set by noise in the simulation results. To visualize the relevance of that incertitude, we observe that the minimum $\gamma^{\mathrm{LR}}(q)$, at $q \approx 0.6 / b$, is $\mathcal{A}^{\mathrm{LR}} /\left(64 \pi e b^{2}\right) \approx 0.01 \epsilon \sigma^{-2}$ below $\gamma^{\mathrm{LR}}(0)$. Therefore, for a realistic representation of a molecular liquid near its triple point, the enhancement due to the ND effect is within a $1 \%$ change in $\gamma(q)$. The much stronger effect $[\gamma(q) / \gamma(0) \approx 0.5]$ reported from some analysis of $\mathrm{x}$-ray diffraction [11-14] could only be obtained with $b \approx 0.2 \sigma$, i.e., 10 times smaller than our result. That implies a difference $\kappa\left(r_{c}\right)-\kappa_{\mathrm{ISM}}\left(r_{c}\right) \approx \mathcal{A}^{\mathrm{LR}} \log (0.1) /(32 \pi) \approx-k T$ in the results for the bending modulus in short-ranged systems, pushing the positive values, $\beta \kappa_{\mathrm{ISM}}\left(r_{c}\right) \approx 0.5$, for $r_{c}=2.5 \sigma$, to $\beta \kappa\left(r_{c}\right) \approx$ -0.5 . Such negative bending moduli are obtained from MD simulations with short-range interactions analyzed with oneparticle definitions for $\xi(\mathbf{x})$, equivalent to the $\mathrm{CW}$ signal in the two-particle correlation from diffraction experiments. We may conclude that such large CW enhancement has little do with the ND effect induced by the LR dispersion forces and that it comes from the lack of sensitivity in the experimental, theoretical, or simulation analysis to separate the bulklike fluctuations near the surface from the capillary waves on the surface.

It is interesting to see how the nonanalytic effect is built up in $\gamma^{\mathrm{LR}}(q)$, from the analytic shapes of $\gamma\left(q ; r_{c}\right)$. Increasing $r_{c}$ the difference to the LR limit becomes smaller and restricted to lower wave vectors. Except for the strict nonanalyticity at $q=$ 0 , any relevant feature of $\gamma^{\mathrm{LR}}(q)$ should already be observed in $\gamma\left(q ; r_{c}\right)$ for $q r_{c} \gtrsim 6$. The strict $r_{c} \rightarrow \infty$ limit is not required to get $\mathrm{CW}$ enhancement and negative bending modulus. The physical cutoff of the dispersion forces from the retardation effects, at $r_{c}^{\text {ret }} \sim 100 \sigma$, would make no difference for $q \gtrsim$ $0.05 / \sigma$. The regular behavior of $\gamma\left(q ; r_{c}\right)$ for any large $r_{c}$ may be seen as a "last minute regularization" of the (nonanalytic) $\gamma^{\mathrm{LR}}(q)$ as $q$ goes to zero.

From (3), (12), and (13) we get the MF-DF prediction for the ND effect on the bending modulus of (regular) truncated LJ liquid surfaces,

$$
\kappa\left(r_{c}\right)=\frac{\mathcal{A}\left(r_{c}\right)}{32 \pi} \log \left(\frac{5.03 b}{r_{c}}\right) .
$$

The nonanalyticity of $\gamma^{\mathrm{LR}}(q)$ is reflected in the $\kappa\left(r_{c}\right) \sim$ $-\log \left(r_{c}\right)$ dependence of the bending modulus of (regular) truncated systems. Notice that any improvement of the DF analysis beyond the sharp-kink approximation would only affect the $\mathcal{O}\left(q^{4}\right)$ terms in (3), with no change in (18). The parameter $b$ may be interpreted in terms of the threshold $r_{c} \geqslant 5.03 b\left(\approx 12 \sigma\right.$ for a LJ liquid at $\left.T^{*}=0.763\right)$ to get $\kappa\left(r_{c}\right)<0$. The decay of our MD-ISM results for $\kappa\left(r_{c}\right) / \mathcal{A}\left(r_{c}\right)$ presented in Fig. 5 is clearly slower than the prediction (18). This discrepancy is explained by the finite-size sampling of the simulations, with $q \geqslant 2 \pi / L_{x}=0.15 / \sigma$. The estimation of $\kappa\left(r_{c}\right)$ from the fit to (3) agrees very well with the (numerical) second derivative of (11) in the range $q \sigma \approx 0.3-0.4$ rather than with the asymptotic behavior $\chi^{\prime \prime}(x) \sim \log (x)$, predicted for $q r_{c} \ll 1$ and used to get (18). 


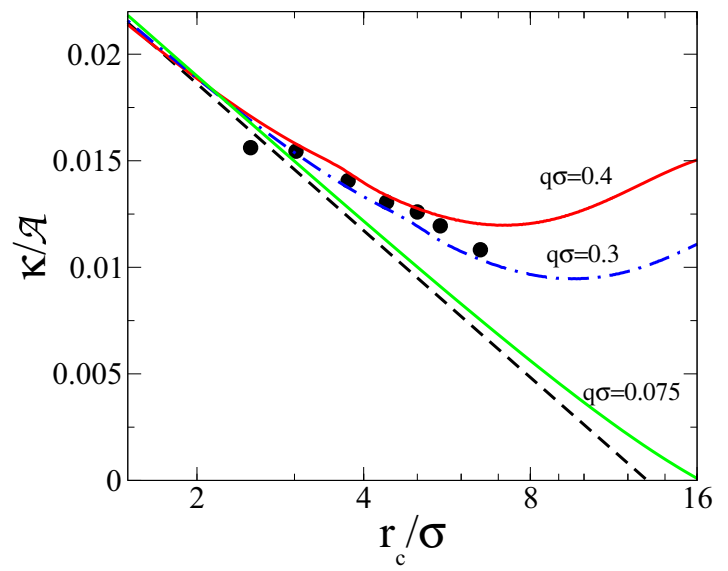

FIG. 5. (Color online) The bending modulus scaled by the Hamaker constant, $\kappa / \mathcal{A}$, as function of the LJ cutoff $r_{c} / \sigma$ (in $\log$ scale). Black circles: MD-ISM results. Black broken line: theoretical DF-MFA prediction (18), with the parameter $b$ from MD-ISM. The other curves are the effective finite-size bending modulus evaluated from the second derivative of $\gamma\left(q ; r_{c}\right)=\gamma^{\mathrm{LR}}(q)-\frac{\mathcal{A}\left(r_{c}\right)}{4 \pi r_{c}^{2}} \chi\left(q r_{c}\right)$ at different finite values of the wave vector: $q \sigma=0.3$ [dashed-dotted (blue) line] and 0.4 [dark solid (red) line] represent typical values in the middle of the interval $\left[2 \pi \sigma / L_{x}, 0.7\right]=[0.15,0.7]$ used to extract $\kappa\left(r_{c}\right)$ in our simulations. The $q \sigma=0.075$ [light solid (green) line] would require a transverse box size $L_{x}=160 \sigma$ to get negative bending modulus for $r_{c} \gtrsim 16 \sigma$.

\section{CONCLUDING REMARKS}

We have presented clear evidence that the nonanalytic enhancement of mesoscopic CW fluctuations predicted by Napiórkowski and Dietrich (ND) [6] is present in a LJ liquid surface. Besides the interest of the direct observation of that peculiar effect in MD simulations, and its quantification for a realistic simple liquid near its triple point temperature, it is most remarkable that we gain that evidence from the matching of two very different approaches: the simplest MF-DF approx- imation for the LR tail of the potential and the percolation (many-particle) analysis of the ISM for the truncated LJ systems in the MD simulations. The computational complexity of the ISM is required to get a good separation between the (CW) surfacelike and the bulklike fluctuations, which cannot be achieved with one-particle definitions of $\xi(\mathbf{x})$, nor with any DF approach based on the one-particle distribution function $\rho[z-\xi(\mathbf{x})]$. The same DF scheme, if used to get the full $\gamma\left(q ; r_{c}\right)$ in systems with short-range interactions, would give monotonically decreasing functions that frustrate the matching between the molecular and the mesoscopic descriptions of the liquid surface. Like the early results of Derjaguin and Hamaker, the robustness of the Napiórkowski-Dietrich effect reflects its essentially geometrical character but applied to the fluctuating shape rather than to the mean regular shape of the liquid phase. The nonanalytic term in (4) depends on the bulk densities through the Hamaker constant $\mathcal{A}^{\mathrm{LR}}$, but it is fully independent of the molecular structure at the interface. That makes it very robust with respect to the definition of the intrinsic surface shape $\xi(\mathbf{x})$ associated to the instantaneous molecular positions. On the other hand, the molecular structure at the interface, and its representation through $\xi(\mathbf{x})$, are crucial to get the shape of $\gamma^{\mathrm{LR}}(q)$. The ISM result for the parameter $b$ provides the quantitative information needed to close the gap between the molecular and the mesoscopic descriptions of fluid interfaces, including the subtle effect of the long-range forces predicted by Napiórkowski-Dietrich. Very recent MD simulations [32] show an interesting dependence of $\gamma\left(q ; r_{c}\right)$ with increasing temperature which should also be investigated by use of our method.

\section{ACKNOWLEDGMENTS}

We acknowledge S. Dietrich, F. Höfling, and M. Napiórkowski for very illuminating discussions and the financial support by the Spanish Ministry of Science and Innovation (Grant No. FIS2010-22047-C05) and the Comunidad Autónoma de Madrid under program MODELICO (Grant No. S2009/ESP-1691).
[1] F. London, Z. Physik 63, 245 (1930).

[2] B. Derjaguin, Kolloid Z. 69, 155 (1934).

[3] H. C. Hamaker, Physica A 10, 1058 (1937).

[4] S. Dietrich and M. Napiórkowski, Phys. Rev. A 43, 1861 (1991).

[5] S. Dietrich, J. Phys. Cond. Mat. 8, 9127 (1996).

[6] M. Napiórkowski and S. Dietrich, Phys. Rev. E 47, 1836 (1993).

[7] J. S. Rowlinson and B. Widom, Molecular Theory of Capillarity (Clarendon Press, London, 1982).

[8] R. Evans, Advan. Phys. 28, 143 (1979).

[9] W. Helfrich, Z. Naturforsch C 28, 693 (1973).

[10] P. Pershan, Colloids Surf. A 171, 149 (2000).

[11] C. Fradin, A. Braslau, D. Luzet, D. Smilgles, M. Alba, N. Boudet, K. Mecke, and J. Daillant, Nature 403, 871 (2000).

[12] S. Mora, J. Daillant, K. Mecke, D. Luzet, A. Braslau, M. Alba, and B. Struth, Phys. Rev. Lett. 90, 216101 (2003).
[13] D. Li, B. Yang, B. Lin, M. Meron, J. Gebhardt, T. Graber, and S. A. Rice, Phys. Rev. Lett. 92, 136102 (2004).

[14] D. Li, X. Jiang, B. Lin, M. Meron, and S.A. Rice, Phys. Rev. B 72, 235426 (2005).

[15] J. Stecki, J. Chem. Phys. 109, 5002 (1998).

[16] R. Vink, J. Horbach, and K. Binder, J. Chem. Phys. 122, 134905 (2005).

[17] K. R. Mecke and S. Dietrich, Phys. Rev. E 59, 6766 (1999).

[18] E. M. Blokhuis, J. Kuipers, and R. L. C. Vink, Phys. Rev. Lett. 101, 086101 (2008).

[19] E. M. Blokhuis, J. Chem. Phys. 130, 014706 (2009).

[20] O. Shpyrko, M. Fukuto, P. Pershan, B. Ocko, I. Kuzmenko, T. Gog, and M. Deutsch, Phys. Rev. B 69, 245423 (2004).

[21] P. Tarazona, R. Checa, and E. Chacón, Phys. Rev. Lett. 99, 196101 (2007).

[22] E. Chacón and P. Tarazona, Phys. Rev. Lett. 91, 166103 (2003). 
[23] P. Tarazona and E. Chacón, Phys. Rev. B 70, 235407 (2004).

[24] F. Stillinger, J. Chem. Phys. 76, 1087 (1982).

[25] J. Chowdhary and B. M. Ladanyi, J. Phys. Chem. B 110, 15442 (2006).

[26] M. Jorge, P. Jedlovszky, and N. Cordeiro, J. Phys. Chem. C 114, 11169 (2010).

[27] M. Jorge, G. Hantal, P. Jedlovszky, and N. Cordeiro, J. Phys. Chem. C 114, 18656 (2010).

[28] A. Willard and D. Chandler, J. Phys. Chem. B 114, 1954 (2010).

[29] D. Zhukhovitskii, J. Chem. Phys. 125, 234701 (2006).

[30] L. Partay, G. Hantal, P. Jedlovszky, A. Vincze, and G. Horvai, J. Compt. Chem. 29, 945 (2008).

[31] R. Delgado-Buscalioni, E. Chacón, and P. Tarazona, Phys. Rev. Lett. 101, 106102 (2008).

[32] F. Höfling and S. Dietrich (private communication).

[33] F. Sedlmeier, D. Horinek, and R. R. Netz, Phys. Rev. Lett. 103, 136102 (2009).
[34] See Supplemental Material at http://link.aps.org/supplemental/ 10.1103/PhysRevE.89.042406 for Intrinsic Sampling Method details.

[35] J. P. Hansen and I. R. McDonald, Theory of Simple Liquids with Applications to Soft Matter (Academic Press, San Diego, CA, 2013), p. 143.

[36] S. J. Plimpton, J. Comp. Phys. 117, 1 (1995).

[37] R. de Gregorio, J. Benet, N. Katcho, F. Blas, and L. MacDowell, J. Chem. Phys. 136, 104703 (2012).

[38] E. Chacón and P. Tarazona, J. Phys.: Condens. Matter 117, S3493 (2005).

[39] E. M. Fernández, E. Chacón, and P. Tarazona, Phys. Rev. B 86, 085401 (2012).

[40] E. Chacón, E. M. Fernández, D. Duque, R. Delgado-Buscalioni, and P. Tarazona, Phys. Rev. B 80, 195403 (2009).

[41] P. Tarazona, E. Chacón, and F. Bresme, J. Chem. Phys. 139, 094902 (2013).

[42] E. M. Fernández, E. Chacón, P. Tarazona, A. O. Parry, and C. Rascón, Phys. Rev. Lett. 111, 096104 (2013). 